\title{
Capsule Commentary on Tannenbaum et al., Nudging Physician Prescription Decisions by Partitioning the Order Set: Results of a Vignette-Based Study
}

\author{
James F. Burgess, Jr., PhD ${ }^{1,2}$, Eric A. Jones, $\mathrm{MPH}^{2}$, and Jake R. Morgan, $\mathrm{MS}^{2}$ \\ ${ }^{1}$ Center for Healthcare Organization and Implementation Research, VA Boston Healthcare System (152 M), Boston, MA, USA; ${ }^{2}$ School of Public \\ Health, Boston University, Boston, MA, USA.
}

J Gen Intern Med 30(3):343

DOI: $10.1007 / \mathrm{s} 11606-014-3095-3$

() Society of General Internal Medicine 2014

$\mathrm{T}$ annenbaum et al. ${ }^{1}$ use vignettes to explore the effect of the order that information is provided in computerized provider order entry (CPOE) on policy-critical practices, such as use of antibiotics. With the proliferation of CPOE systems, a number of advantages associated with their use have been revealed: reducing errors caused by misreading handwriting, reducing duplicate or incorrect doses or tests, and alerting providers to potential medication interactions. In this value-focused environment, administrators have become much more highly capable and willing to make design and implementation decisions that may affect clinical practice.

This process change is attractive in its relative ease of implementation and dissemination, given the difficulty in changing antibiotic prescribing behavior. However, success will depend on effective integration into existing workflow. Clinicians are known to alter quality and safety measures in order to expedite treatment or address patient concerns. These "workarounds" have been studied in the context of healthcare administration safeguards, ${ }^{2}$ and their effect on the subversion of medication safety measures illustrates likely challenges to EMR design changes motivated by this work.

The problems of workarounds as well as shortcuts reflect underlying forces behind the change in the behavior studied. CPOE users employ a variety of "shortcuts" to increase efficiency and reduce search costs. The authors suggest that new ordering could be a shortcut for signaling which choices are "common or appropriate". Future research on inappropriate use should grapple directly with when and why providers employ shortcuts. The shortcut may dictate that it is "safer" to prescribe, given uncertainty, that it is easier to quiet a demanding patient with a prescription, or that it is faster to write a script and move to the next appointment.
Understanding how to increase the perceived "cost" of overprescribing such that risks are $\operatorname{stark}^{3}$ and framing guidelines as worthwhile investments of time is critical. If CPOE modifications are not matched with parallel alterations in underlying clinician knowledge, attitudes, and behaviors, the effects may be temporary and may fall short of the goal of significant and long-lasting reductions in inappropriate antibiotic utilization. The inclusion of user input in CPOE design and the study of the impact on work practices of prescribing providers warrants more effort and attention.

\begin{abstract}
Acknowledgments: This project was supported in part by the Jayne Koskinas Ted Giovanis Foundation for Health and Policy, a private foundation based in Highland, Maryland, dedicated to effecting change in the health care industry for the greater public good. The opinions, findings, and conclusions or recommendations expressed in this material are those of the authors, and not necessarily those of the Jayne Koskinas Ted Giovanis Foundation for Health and Policy, its directors, officers, or staff. This project was also supported in part by grant number T32HSO22242 from the Agency for Healthcare Research and Quality. The content is solely the responsibility of the authors, and does not necessarily represent the official views of the Agency for Healthcare Research and Quality. The views expressed in this article are those of the authors and do not necessarily reflect the position or policy of Boston University, the Department of Veterans Affairs, or the United States government.
\end{abstract}

Conflict of Interest: The authors have no conflicts regarding any of the material in this manuscript.

Corresponding Author: James F. Burgess, Jr., PhD; Center for Healthcare Organization and Implementation Research, VA Boston Healthcare System (152 M), 150 South Huntington Avenue, Boston, MA 02130, USA (e-mail: james.burgess@va.gov).

\section{REFERENCES}

1. Tannenbaum D, Doctor JN, Persell SD, Friedberg MW, Meeker D, Friesema EM, Goldstein NJ, Linder JA, Fox CR. nudging physician prescription decisions by partitioning the order set: results of a vignettebased study. J Gen Intern Med. doi:10.1007/s11606-014-3051-2

2. Koppel RP, Wetterneck TM, Telles JL, Karsh B-T. Workarounds to Barcode Medication Administration Systems: Their Occurences, Causes, and Threats to Patient Safety. J Am Med Inform Assoc. 2008;15(4):408-421.

3. Cabana MD, Rand CS, Powe NR, et al. Why don't physicians follow clinical practice guidelines? A framework for improvement. JAMA. 1999;282(15):1458-1465. doi:10.1001/jama.282.15.1458. 